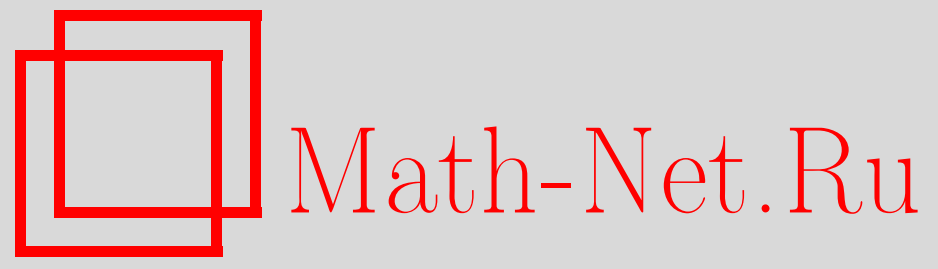

А. Н. Тимашёв, Закон больших чисел для перманентов случайных матриц, Дискрет. матем., 2005, том 17, выпуск 4, 59-71

DOI: https://doi.org/10.4213/dm129

Использование Общероссийского математического портала Math-Net.Ru подразумевает, что вы прочитали и согласны с пользовательским соглашением http://www . mathnet.ru/rus/agreement

Параметры загрузки:

IP : 35.174 .16 .151

26 апреля 2023 г., 09:59:13

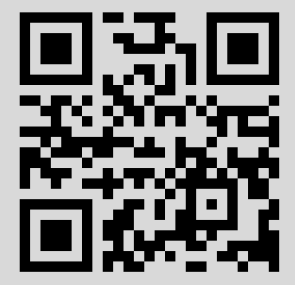


УдК 519.2

\title{
Закон больших чисел для перманентов случайных матриц
}

\author{
(ㄷ) 2005 г. А. Н. Тимашёв
}

\begin{abstract}
Рассматривается класс случайных матриц $C=\left(c_{i j}\right), i, j=1, \ldots, N$, элементы которых - независимые случайные величины, имеющие одинаковое распределение, совпадающее с распределением случайной величины $\xi$, для которой $E \xi^{2}>0$. Как обычно, $\operatorname{per} C$ обозначает перманент матрицы $C$. В схеме серий, когда $\xi=\xi_{N}, \mathbf{E} \xi_{N} \neq 0$ при $N=1,2, \ldots$ и $\mathrm{D} \xi_{N}=o\left(\left(\mathrm{E} \xi_{N}\right)^{2}\right)$ при $N \rightarrow \infty$, доказывается, что последовательность случайных величин $\operatorname{per} C /\left(N !\left(\mathbf{E} \xi_{N}\right)^{N}\right)$ сходится по вероятности к единице при $N \rightarrow \infty$. Аналогичный результат устанавливается и в более общем случае, когда строки матрицы $C$ - независимые $N$-мерные случайные векторы, имеющие одинаковое распределение, совпадающее с распределением некоторого случайного вектора $\mu$, компоненты которого одинаково распределены, но, вообще говоря, зависимы. Приводятся достаточные условия справедливости закона больших чисел для последовательности $\operatorname{per} C / \mathrm{E}$ per $C$ в случае, когда вектор $\mu$ совпадает с вектором частот исходов равновероятной полиномиальной схемы с $N$ исходами и $n$ испытаниями, а также в предположении, что $\mu$-случайное равновероятное решение уравнения $k_{1}+\ldots+k_{N}=n$ в целых неотрицательных числах $k_{1}, \ldots, k_{N}$.
\end{abstract}

Рассмотрим класс неотрицательных $N \times N$ матриц $A=\left(a_{i j}\right), i, j=1, \ldots, N$, строки которых - независимые $N$-мерные случайные величины, распределение которых совпадает с распределением вектора $\left(\xi_{1}, \ldots, \xi_{N}\right)$ частот исходов равновероятной полиномиальной схемы с $N$ исходами и $n$ испытаниями, $n, N \geqslant 2$. Тогда $\xi_{1}+\ldots+\xi_{N}=n$ и $\mathbf{E} \xi_{i}=n / N$, $i=1, \ldots, N$.

Пусть $\operatorname{per} A$ обозначает перманент матрищы $A$, тогда

$$
\mathrm{E} \operatorname{per} A=\sum_{\left(i_{1}, \ldots, i_{N}\right)} \mathrm{E} a_{1 i_{1}} \ldots \mathrm{E} a_{N i_{N}}=N !\left(\frac{n}{N}\right)^{N},
$$

где суммирование осуществляется по всем $N$ ! перестановкам $\left(i_{1}, \ldots, i_{N}\right)$ чисел $1, \ldots, N$.

Для подсчета второго момента $\mathrm{E}(\operatorname{per} A)^{2}$ используем метод, по-видимому, впервые реализованный O'Нейлом (см., например, [1], с. 80). Известно (см., например, [2], с. 471), что для $i, j=1, \ldots, N, i \neq j$,

$$
\mathbf{E}\left(\xi_{i} \xi_{j}\right)=\frac{n(n-1)}{N^{2}}, \quad \mathbf{E} \xi_{i}^{2}=\frac{n(n-1)}{N^{2}}+\frac{n}{N} .
$$

Из равенства

$$
\operatorname{per} A=\sum_{\left(i_{1}, \ldots, i_{N}\right)} a_{1 i_{1}} \ldots a_{N i_{N}}
$$


следует, что

$$
\mathrm{E}(\operatorname{per} A)^{2}=\sum_{\left(i_{1}, \ldots, i_{N}\right),\left(j_{1}, \ldots, j_{N}\right)} \mathrm{E}\left(a_{1 i_{1}} a_{1 j_{1}}\right) \ldots \mathrm{E}\left(a_{N i_{N}} a_{N j_{N}}\right)
$$

где суммирование осуществляется по всем $(N !)^{2}$ упорядоченным парам перестановок $\left(i_{1}, \ldots, i_{N}\right)$ и $\left(j_{1}, \ldots, j_{N}\right)$ чисел $1, \ldots, N$.

Пусть $m$ - расстояние между соответствующими подстановками, то есть

$$
m=\operatorname{card}\left\{v \mid 1 \leqslant v \leqslant N, i_{\nu} \neq j_{v}\right\}
$$

Известно (см. [1], с. 80), что число всевозможных подстановок, находящихся на расстояния $m$ от любой фиксированной подстановки степени $N$, равно

$$
(N)_{m} S_{m}=\frac{N !}{(N-m) !} S_{m}, \quad m=0,1, \ldots, N
$$

где

$$
S_{m}=\sum_{k=0}^{m} \frac{(-1)^{k}}{k !}, \quad m=0,1, \ldots
$$

Из соотношений (2)-(4) получаем, что

$$
\mathbf{E}(\operatorname{per} A)^{2}=N ! \sum_{m=0}^{N}(N)_{m} S_{m}\left(\mathbf{E}\left(\xi_{1} \xi_{2}\right)\right)^{m}\left(\mathbf{E} \xi_{1}^{2}\right)^{N-m}
$$

Подставляя в (6) значения $\mathbf{E}\left(\xi_{1} \xi_{2}\right)$ и $\mathbf{E} \xi_{1}^{2}$ из (2), приходим к равенству

$$
\mathrm{E}(\operatorname{per} A)^{2}=N !\left(\frac{n}{N}\right)^{N}\left(1+\frac{n-1}{N}\right)^{N} \sum_{m=0}^{N}(N)_{m} S_{m}\left(\frac{n-1}{N+n-1}\right)^{m}
$$

В настоящей работе с использованием соотношений (1), (5), (7) доказывается закон больших чисел для перманента случайной матрицы $A$, аналогичный законам больших чисел для перманента случайных $(0,1)$-матриц с заданным числом единиц и с заданными суммами элементов по строкам и столбцам, доказанным в [3, 4] (см. также [1]). Ранее похожие задачи рассматривались в [5] для классов случайных $(0,1)$-матриц с заданным числом единиц в каждой строке и случайных стохастических матриц с независимыми одинаково распределенными строками, имеющими симметричное распределение Дирихле.

Заметим, что если каждый элемент матрицы $A$ разделить на $n$, то получится случайная стохастическая матрища $A^{\prime}$, для которой согласно (1) и (7)

$$
\mathrm{E}\left(\operatorname{per} A^{\prime}\right)=N ! N^{-N}, \quad \mathbf{E}\left(\operatorname{per} A^{\prime}\right)^{2}=n^{-2 N} \mathbf{E}(\operatorname{per} A)^{2} .
$$

Далее рассматривается класс неотрицательных $N \times N$ матриц

$$
B=\left(b_{i j}\right), \quad i, j=1, \ldots, N
$$


строки которых - независимые $N$-мерные случайные величины. Предполагается, что распределение каждой из них совпадает с распределением случайного вектора $\left(\varkappa_{1}, \ldots, \varkappa_{N}\right)$ такого, что

$$
\mathbf{P}\left\{\varkappa_{1}=k_{1}, \ldots, \varkappa_{N}=k_{N}\right\}=\left(\begin{array}{c}
n+N-1 \\
N-1
\end{array}\right)^{-1}
$$

при любом выборе упорядоченного набора $\left(k_{1}, \ldots, k_{N}\right)$ неотрицательных целых чисел, для которых $k_{1}+\ldots+k_{N}=n, n, N \geqslant 2$. Тогда $\varkappa_{1}+\ldots+\varkappa_{N}=n$ и Е $\varkappa_{i}=n / N$, $i=1, \ldots, N$. Поэтому аналогично (1)

$$
\text { E per } B=N !\left(\frac{n}{N}\right)^{N}
$$

Нетрудно показать, что при $i, j=1, \ldots, N, i \neq j$,

$$
\mathbf{E}\left(\varkappa_{i} \varkappa_{j}\right)=\frac{n(n-1)}{N(N+1)}, \quad \mathbf{E} \varkappa_{i}^{2}=\frac{n(2 n+N-1)}{N(N-1)} .
$$

Используя (6) с заменой $\mathbf{E}\left(\xi_{1} \xi_{2}\right)$ и $\mathbf{E} \xi_{1}^{2}$ на $\mathbf{E}\left(\varkappa_{1} \varkappa_{2}\right)$ и $\mathbf{E} \varkappa_{1}^{2}$ и учитывая (9), получаем, что

$$
\mathrm{E}(\operatorname{per} B)^{2}=N !\left(\frac{n}{N}\right)^{N}\left(1+\frac{2(n-1)}{N-1}\right)^{N} \sum_{m=0}^{N}(N)_{m} S_{m}\left(\frac{n-1}{2 n+N-1}\right)^{m} .
$$

С использованием формул (5), (8), (10) в работе доказывается закон больших чисел для перманента случайной матрищы $B$.

Заметим, что если каждый элемент матрищы $B$ разделить на $n$, то получится случайная стохастическая матрища $B^{\prime}$, для которой согласно (8) и (10)

$$
\text { E per } B^{\prime}=N ! N^{-N}, \quad \mathbf{E}\left(\operatorname{per} B^{\prime}\right)^{2}=n^{-2 N} \mathbf{E}(\operatorname{per} B)^{2} \text {. }
$$

Лемма 1. Справедливо равенство

$$
\mathrm{E}(\operatorname{per} A)^{2}=(N !)^{2}\left(\frac{n(n-1)}{N^{2}}\right)^{N} \sum_{m=0}^{N} \frac{1}{m !}\left(\frac{N}{n-1}\right)^{m} .
$$

Доказательство. В [5] установлено, что при $0<p \leqslant 1$

$$
\sum_{m=0}^{N}(N)_{m} S_{m} p^{m}=N ! p^{N} \sum_{m=0}^{N} \frac{\left(p^{-1}-1\right)^{m}}{m !}
$$

Полагая $p=(n-1) /(N+n-1)$ и учитывая (7) и (12), получаем (11). Лемма 1 доказана.

Лемма 2. $\Pi p u n \rightarrow \infty u N=o(n)$

$$
\mathrm{E}(\operatorname{per} A)^{2}=(N !)^{2} N^{-2 N}(n(n-1))^{N}(1+O(N / n)) .
$$

Доказательство. При выполнении условий леммы

$$
\sum_{m=0}^{N} \frac{(N /(n-1))^{m}}{m !}=1+O(N / n)
$$

Подставляя эту оценку в (11), получаем (13). Лемма 2 доказана. 
Замечание 1. Если при выполнении условий леммы 2 параметр $N \rightarrow \infty$, то

$$
\mathrm{E}(\operatorname{per} A)^{2}=2 \pi N\left(n e^{-1}\right)^{2 N}(1+O(N / n)+O(1 / N)) .
$$

Лемма 3. Пусть $n, N \rightarrow \infty$ так, что

$$
0<\alpha_{0} \leqslant \alpha=n / N \leqslant \alpha_{1},
$$

где $\alpha_{0}, \alpha_{1}$ - постоянные. Тогда равномерно относительно $\alpha \in\left[\alpha_{0}, \alpha_{1}\right]$

$$
\mathrm{E}(\operatorname{per} A)^{2}=2 \pi N\left(n e^{-1}\right)^{2 N}(1+O(1 / N)) .
$$

Доказательство. При условиях (14) равномерно относительно $\alpha \in\left[\alpha_{0}, \alpha_{1}\right]$

$$
(N !)^{2} N^{-2 N}(n(n-1))^{N}=2 \pi N(n / e)^{2 N} e^{-1 / \alpha}(1+O(1 / N)) .
$$

Кроме того,

$$
\sum_{m=0}^{N} \frac{(N /(n-1))^{m}}{m !}=e^{1 / \alpha}(1+O(1 / N))
$$

равномерно в указанном выше смысле.

Из двух последних соотношений с учетом (11) следует оценка (15). Лемма 3 доказана.

Замечание 2. Леммы 2 и 3 в совокупности означают, что при $n, N \rightarrow \infty$ и $N=O(n)$

$$
\mathrm{E}(\operatorname{per} A)^{2}=2 \pi N\left(n e^{-1}\right)^{2 N}(1+o(1)) .
$$

Замечание 3. Если $n=2$, то при $N \rightarrow \infty$ из (11) и равенства

$$
\sum_{m=0}^{N} \frac{N^{m}}{m !}=\frac{1}{2} e^{N}(1+o(1))
$$

следует, что

$$
\mathrm{E}(\operatorname{per} A)^{2}=\pi N\left(\frac{2}{e}\right)(1+o(1)) .
$$

Лемма 4. При $N \rightarrow \infty, n=o(N), n \geqslant 3$

$$
\mathrm{E}(\operatorname{per} A)^{2}=2 \pi N\left(n(n-1) e^{-2}\right)^{N} e^{N /(n-1)}(1+o(1)) .
$$

Доказательство. Используя формулу Стирлинга и учитывая (11), получаем, что

$$
\mathbf{E}(\operatorname{per} A)^{2}=2 \pi N\left(n(n-1) e^{-2}\right)^{N} \sum_{m=0}^{N} \frac{(N /(n-1))^{m}}{m !}(1+O(1 / N)) .
$$

Пусть $\lambda=N /(n-1) \rightarrow \infty$ и $\chi-$ случайная величина, распределенная по закону Пуассона с параметром $\lambda$. Тогда

$$
\sum_{m=0}^{N} \frac{(N /(n-1))^{m}}{m !}=e^{N /(n-1)} \mathbf{P}\{(\chi-\lambda) / \sqrt{\lambda} \leqslant(n-2) \sqrt{\lambda}\}=e^{N /(n-1)}(1+o(1))
$$

Из этой оценки и (18) получаем (17). Лемма 4 доказана. 
Замечание 4. При условиях леммы 4 из (1) и (17) следует, что

$$
\ln \mathrm{E}\left(\frac{\operatorname{per} A}{\mathrm{E} \operatorname{per} A}\right)^{2}=N(\ln (1-1 / n)+1 /(n-1))+o(1) .
$$

Теорема 1. Пусть $n \rightarrow \infty u N=N(n)$ меняется так, ито $N=o\left(n^{2}\right), N \geqslant 2$. Тогда последовательность случайных величин

$$
\eta_{n}=\frac{\operatorname{per} A}{\mathrm{E} \operatorname{per} A}, \quad n=2,3, \ldots,
$$

сходится по вероятности к единице.

Доказательство. Если $n \rightarrow \infty$ и $N \geqslant 2$ фиксировано, то согласно (1) и (13)

$$
\left.\mathrm{E} \eta_{n}^{2}=\frac{\mathrm{E}(\operatorname{per} A)^{2}}{(\mathrm{E} \operatorname{per} A)^{2}}=\left(1-\frac{1}{n}\right)^{N}(1+O(1 / n))=1+O(1 / n)\right) .
$$

Если же $N \rightarrow \infty$ и $N=O(n)$, то из (1) и (16) получаем, что

$$
E \eta_{n}^{2}=1+o(1) \text {. }
$$

Если, наконец, при $n \rightarrow \infty$ и $N=N(n) \rightarrow \infty, n=o(N)$ и $N=o\left(n^{2}\right)$, то из (19) и соотношения

$$
\ln \left(1-\frac{1}{n}\right)+\frac{1}{n-1}=\frac{1}{2 n^{2}}(1+O(1 / n))
$$

следует, что оценка (20) выполняется. Утверждение теоремы следует из неравенства Чебышева, поскольку $\mathbf{E} \eta_{n}=1, n=1,2, \ldots$, и $\mathbf{D} \eta_{n}=o(1)$ при $n \rightarrow \infty$.

Теорема 1 доказана.

Замечание 5. Если $n, N \rightarrow \infty$ так, что $N n^{-2} \rightarrow \beta, 0<\beta<\infty$, то согласно (19)

$$
\mathrm{E}(\operatorname{per} A)^{2}=(\mathrm{E} \operatorname{per} A)^{2} e^{\beta / 2}(1+o(1)) .
$$

Если же $n, N \rightarrow \infty$ так, что $N n^{-2} \rightarrow \infty$, то

$$
(\mathrm{E} \operatorname{per} A)^{2}=o\left(\mathrm{E}(\operatorname{per} A)^{2}\right) .
$$

Лемма 5. Справедливо равенство

$$
\mathbf{E}(\operatorname{per} B)^{2}=(N !)^{2}\left(\frac{n(n-1)}{N(N+1)}\right)^{N} \sum_{m=0}^{N} \frac{((n+N) /(n-1))^{m}}{m !} .
$$

Доказательство. Формула (21) следует из (10) и (12), если положить

$$
p=(n-1) /(2 n+N-1) \text {. }
$$

Лемма 5 доказана.

Лемма 6. При $n \rightarrow \infty$ и фиксированном $N \geqslant 2$

$$
\mathrm{E}(\operatorname{per} B)^{2}=(N !)^{2}\left(\frac{n}{N}\right)^{2 N}\left(1-\frac{1}{N+1}\right)^{N} \sum_{m=0}^{N} \frac{1}{m !}(1+O(1 / n)) .
$$


Доказательство. При выполнении условий леммы 6

$$
\sum_{m=0}^{N} \frac{((n+N) /(n-1))^{m}}{m !}=\sum_{m=0}^{N} \frac{1}{m !}(1+O(1 / n)) .
$$

Подставляя эту оценку в (21) и проводя элементарные преобразования, получаем соотношение (22). Лемма 6 доказана.

Лемма 7. $\Pi p u n, N \rightarrow \infty u N=o(n)$

$$
\mathbf{E}(\operatorname{per} B)^{2}=2 \pi N(n / e)^{2 N}(1+o(1)) \text {. }
$$

Доказательство. Оценка (23) получается из (21) с использованием формулы Стирлинга, если учесть, что в рассматриваемом случае

$$
\sum_{m=0}^{N} \frac{((n+N) /(n-1))^{m}}{m !}=e(1+o(1)) .
$$

Лемма 7 доказана.

Лемма 8. При выполнении условий леммы 3 справедлива оченка (23).

Доказательство соотношения (23) проводится аналогично доказательству справедливости оценки (15).

Замечание 6. Леммы 7 и 8 в совокупности означают, что при $n, N \rightarrow \infty$ и $N=O(n)$ оценка (23) остается справедливой.

Замечание 7. Если $n=2$, то при $N \rightarrow \infty$ из (21) и равенства

$$
\sum_{m=0}^{N} \frac{(N+2)^{m}}{m !}=\frac{1}{2} e^{N+2}(1+o(1))
$$

следует, что

$$
\mathrm{E}(\operatorname{per} B)^{2}=\pi e N(2 / e)^{N}(1+o(1)) .
$$

Лемма 9. При выполнении условий леммы 4

$$
\mathbf{E}(\operatorname{per} B)^{2}=2 \pi N\left(n(n-1) e^{-2}\right)^{N} e^{(N+1) /(n-1)}(1+o(1)) .
$$

Доказательство соотношения (24) проводится аналогично доказательству справедливости оценки (17), если положить $\lambda=(n+N) /(n-1)$.

Замечание 8. При выполнении условий леммы 9 из (8) и (24) следует, что

$$
\ln \mathbf{E}\left(\frac{\operatorname{per} B}{\mathrm{E} \operatorname{per} B}\right)^{2}=N\left(\ln \left(1-\frac{1}{n}\right)+\frac{1}{n-1}\right)+\frac{1}{n-1}+o(1) .
$$

Теорема 2. Пусть $n, N \rightarrow \infty$ так, что $N=o\left(n^{2}\right)$. Тогда последовательность случайных величин

$$
\theta_{n}=\frac{\operatorname{per} B}{\mathbf{E} \operatorname{per} B}, \quad n=2,3, \ldots,
$$

сходится по вероятности к единице. 
Доказательство. Если $n, N \rightarrow \infty$ так, что $N=O(n)$, то согласно (8) и замечанию 6

$$
\mathbf{E} \theta_{n}^{2}=1+o(1) \text {. }
$$

Если же $n, N \rightarrow \infty$ так, что $n=o(N)$ и $N=o\left(n^{2}\right)$, то из (25) следует, что оценка (26) остается справедливой. Поэтому при выполнении условий теоремы 2 равенство (26) выполняется, то есть $\mathbf{D} \theta_{n} \rightarrow 0$. Теорема 2 доказана.

Замечание 9. Если $n, N \rightarrow \infty$ так, что $N n^{-2} \rightarrow \beta, 0<\beta<\infty$, то

$$
\mathbf{E} \theta_{n}^{2}=e^{\beta / 2}(1+o(1)),
$$

то есть

$$
\mathbf{E}(\operatorname{per} B)^{2}=(\mathbf{E} \operatorname{per} B)^{2} e^{\beta / 2}(1+o(1)) .
$$

Если же $n, N \rightarrow \infty$ так, что $N n^{-2} \rightarrow \infty$, то

$$
(\mathbf{E} \operatorname{per} B)^{2}=o\left(\mathbf{E}(\operatorname{per} B)^{2}\right) .
$$

Замечание 10. Если $N \geqslant 2$ фиксировано, то

$$
\lim _{n \rightarrow \infty} \mathbf{E} \theta_{n}^{2}=\left(1-\frac{1}{N+1}\right)^{N} \sum_{m=0}^{N} \frac{1}{m !}>1,
$$

поэтому последовательность случайных величин $\theta_{n}, n=2,3, \ldots$, не удовлетворяет закону больших чисел. Так, например, при $N=2$, используя тот факт, что $\varkappa_{1} / n \rightarrow \xi$ по вероятности при $n \rightarrow \infty$, где $\xi$ имеет равномерное распределение на отрезке [0,1], нетрудно убедиться в том, что последовательность случайных величин $n^{-2}$ per $B$ имеет в пределе сосредоточенное на отрезке $[0,1]$ распределение, совпадающее с распределением суммы $\xi+\eta-2 \xi \eta$, где случайные величины $\xi$ и $\eta$ независимы и одинаково распределены. Это означает, что при $N=2$

$$
\lim _{n \rightarrow \infty} \mathbf{P}\left\{n^{-2} \text { per } B<x\right\}= \begin{cases}1 / 2, & \text { если } x=1 / 2, \\ x+(1 / 2-x) \ln |1-2 x|, & \text { если } x \in[0,1 / 2) \cup(1 / 2,1] .\end{cases}
$$

Далее рассматривается класс случайных матриц вида $C=\left(c_{i j}\right), i, j=1, \ldots, N$, где элементы $c_{i j}$ - независимые случайные величины, имеющие одинаковое распределение, совпадающее с распределением случайной величины $\xi$, для которой $\mathbf{E} \xi^{2}>0$. Заменяя в (6) матрищу $A$ на $C, \mathbf{E} \xi_{1}^{2}$ на $\mathbf{E} \xi^{2}$ и $\mathbf{E}\left(\xi_{1} \xi_{2}\right)$ на $(\mathbf{E} \xi)^{2}$, получаем равенство

$$
\mathbf{E}(\operatorname{per} C)^{2}=N !\left(\mathbf{E} \xi^{2}\right)^{N} \sum_{m=0}^{N}(N)_{m} S_{m} p^{m}
$$

где $p=(\mathbf{E} \xi)^{2}\left(\mathbf{E} \xi^{2}\right)^{-1} \in[0,1]$.

Если $\mathrm{E} \xi \neq 0$, то $p>0$. В этом случае из (12) следует равенство

$$
\mathbf{E}(\operatorname{per} C)^{2}=(N !)^{2}(\mathbf{E} \xi)^{2 N} \sum_{m=0}^{N} \frac{\gamma^{m}}{m !}
$$

3 Дискретная математика, т.17 №4 
где

$$
\gamma=p^{-1}-1=\mathbf{D} \xi(\mathbf{E} \xi)^{-2}
$$

Поскольку

$$
\mathbf{E} \operatorname{per} C=N !(\mathbf{E} \xi)^{N}
$$

согласно (27)

$$
\mathbf{E}(\operatorname{per} C)^{2}=(\mathrm{E} \operatorname{per} C)^{2} \sum_{m=0}^{N} \frac{\gamma^{m}}{m !}
$$

Полагая

$$
\chi_{N}=\frac{\operatorname{per} C}{\mathrm{E} \operatorname{per} C}, \quad N=1,2, \ldots,
$$

получаем, что $\mathbf{E}_{\chi_{N}}=1$ и согласно (29)

$$
\mathbf{E}_{N}^{2}=\sum_{m=0}^{N} \frac{\gamma^{m}}{m !}
$$

где значение $\gamma$ определено в (28).

Если же $\mathbf{E} \xi=0$, то

$$
\mathbf{E} \operatorname{per} C=0, \quad \mathbf{E}(\operatorname{per} C)^{2}=N !\left(\mathbf{E} \xi^{2}\right)^{N} .
$$

Будем рассматривать схему серий, когда $\xi=\xi_{N}, N=1,2, \ldots$ В этом случае

$$
\gamma=\gamma_{N}=\mathbf{D} \xi_{N}\left(\mathbf{E} \xi_{N}\right)^{-2}
$$

если только $\mathbf{E} \xi_{N} \neq 0, N=1,2, \ldots$

Теорема 3. Пусть $\mathrm{E} \xi_{N} \neq 0, \mathrm{D} \xi_{N}>0$, и пусmь

$$
\beta_{N}=\frac{\left|\mathbf{E} \xi_{N}\right|}{\sqrt{\mathbf{D} \xi_{N}}}\left(N-\mathbf{D} \xi_{N}\left(\mathbf{E} \xi_{N}\right)^{-2}\right), \quad N=1,2, \ldots
$$

Если $\beta_{N} \rightarrow \infty$ при $N \rightarrow \infty$, то

$$
\mathbf{E}(\operatorname{per} C)^{2}=(N !)^{2}(\mathbf{E} \xi)^{2 N} \exp \left\{\mathbf{D} \xi_{N}\left(\mathbf{E} \xi_{N}\right)^{-2}\right\}(1+o(1))
$$

Eсли $\lim _{N \rightarrow \infty} \beta_{N}=x \in \mathbf{R}$, mo

$$
\mathbf{E}(\operatorname{per} C)^{2}=(N !)^{2}(\mathbf{E} \xi)^{2 N} \exp \left\{\mathbf{D} \xi_{N}\left(\mathbf{E} \xi_{N}\right)^{-2}\right) \Phi(x)(1+o(1)),
$$

где $\Phi(x)$ - функция распределения стандартного нормального закона.

Если же $\beta_{N} \rightarrow-\infty$ при $N \rightarrow \infty$, то

$$
(N !)^{-2}(\mathbf{E} \xi)^{-2 N} \mathbf{E}(\operatorname{per} C)^{2}=o\left(\exp \left\{\mathbf{D} \xi_{N}\left(\mathbf{E} \xi_{N}\right)^{-2}\right\}\right)
$$


Доказательство. Согласно (28)

$$
\gamma=\gamma_{N}=\mathbf{D} \xi_{N}\left(\mathbf{E} \xi_{N}\right)^{-2},
$$

и поэтому

$$
\beta_{N}=\left(\gamma_{N}\right)^{-1 / 2}\left(N-\gamma_{N}\right), \quad N=1,2, \ldots
$$

Если последовательность $\left\{\gamma_{N}\right\}$ ограничена, то из равенства

$$
\sum_{m=0}^{N} \frac{\gamma_{N}^{m}}{m !}=e^{\gamma_{N}}+O\left(\gamma_{N}^{N+1} /(N+1) !\right)
$$

получаем, что

$$
\sum_{m=0}^{N} \frac{\gamma_{N}^{m}}{m !}=e^{\gamma_{N}}(1+o(1))
$$

и оценка (32) следует из (29) и (35).

Пусть $\gamma_{N} \rightarrow \infty$ при $N \rightarrow \infty$. Если случайная величина $\delta_{N}$ имеет распределение Пуассона с параметром $\gamma_{N}$, то

$$
\sum_{m=0}^{N} \frac{\gamma_{N}^{m}}{m !}=e^{\gamma_{N}} \mathbf{P}\left\{\delta_{N} \leqslant N\right\}=e^{\gamma_{N}} \mathbf{P}\left\{\left(\gamma_{N}\right)^{-1 / 2}\left(\delta_{N}-\gamma_{N}\right) \leqslant \beta_{N}\right\} .
$$

Если $\beta_{N} \rightarrow \infty$ при $N \rightarrow \infty$, то

$$
\mathbf{P}\left\{\left(\gamma_{N}\right)^{-1 / 2}\left(\delta_{N}-\gamma_{N}\right) \leqslant \beta_{N}\right\}=1+o(1),
$$

если же $\lim _{N \rightarrow \infty} \beta_{N}=x \in \mathbf{R}$, то

$$
\mathbf{P}\left\{\left(\gamma_{N}\right)^{-1 / 2}\left(\delta_{N}-\gamma_{N}\right) \leqslant \beta_{N}\right\}=\Phi(x)(1+o(1)) .
$$

Если, наконец, $\beta_{N} \rightarrow-\infty$ при $N \rightarrow \infty$, то

$$
\mathbf{P}\left\{\left(\gamma_{N}\right)^{-1 / 2}\left(\delta_{N}-\gamma_{N}\right) \leqslant \beta_{N}\right\}=o(1) .
$$

Оценки (32)-(34) следуют из этих соотношений и формул (29), (35), (36). Теорема 3 доказана.

Замечание 11. При условии, что $\beta_{N} \rightarrow-\infty$ при $N \rightarrow \infty$, оценка (34) в общем случае неулучшаема.

Теорема 4. Пусть $\mathbf{E} \xi_{N} \neq 0, N=1,2, \ldots, u \mathbf{D} \xi_{N}=o\left(\left(\mathbf{E} \xi_{N}\right)^{2}\right) n p u N \rightarrow \infty$. Тогдa последовательность случайных величин

$$
\chi_{N}=\frac{\operatorname{per} C}{N !\left(\mathbf{E} \xi_{N}\right)^{N}}, \quad N=1,2, \ldots,
$$

сходится по вероятности к единиче. 
Доказательство. При сформулированных условиях

$$
\gamma_{N}=\mathbf{D} \xi_{N}\left(\mathbf{E} \xi_{N}\right)^{-2}=o(1)
$$

и поэтому согласно (30) при $\gamma=\gamma_{N}$

$$
\mathbf{E} \chi_{N}^{2}=\sum_{m=0}^{N} \frac{\gamma_{N}^{m}}{m !}=1+o(1),
$$

то есть $\mathrm{D}_{\chi_{N}}=o(1)$ при $N \rightarrow \infty$, и утверждение теоремы 4 следует из неравенства Чебышева.

Замечание 12. Если $\mathrm{E} \xi_{N}=0$, то $\mathrm{E} \operatorname{per} C=0$ и согласно (31)

$$
\mathrm{D} \operatorname{per} C=\mathbf{E}(\operatorname{per} C)^{2}=N !\left(\mathbf{E} \xi_{N}^{2}\right)^{N},
$$

так что при условии, что $N !\left(\boldsymbol{E}_{N}^{2}\right)^{N} \rightarrow 0$ при $N \rightarrow \infty$, последовательность реr $C$, $N=1,2, \ldots$, сходится по вероятности к нулю.

Проиллюстрируем теоремы 3 и 4 на примере случайной величины $\xi$, имеющей равномерное распределение на отрезке $[1, b]$. В схеме серий $\xi=\xi_{N}$ и $a=a_{N}<b=b_{N}$, $N=1,2, \ldots$ Если, кроме того, $a_{N}+b_{N} \neq 0, N=1,2, \ldots$, то при $N \rightarrow \infty$ согласно (27) и (30)

$$
\mathbf{E}(\operatorname{per} C)^{2}=(N !)^{2}\left(\frac{a_{N}+b_{N}}{2}\right)^{2 N} \exp \left\{\frac{\left(a_{N}-b_{N}\right)^{2}}{3\left(a_{N}+b_{N}\right)^{2}}\right\}(1+o(1)) .
$$

Действительно, при таких условиях

$$
\mathbf{E} \xi_{N}=\frac{1}{2}\left(a_{N}+b_{N}\right), \quad \mathbf{E} \operatorname{per} C=N !\left(\mathbf{E} \xi_{N}\right)^{N}, \quad \frac{\mathbf{D} \xi_{N}}{\left(\mathbf{E} \xi_{N}\right)^{2}}=\frac{\left(a_{N}-b_{N}\right)^{2}}{3\left(a_{N}+b_{N}\right)^{2}}, \quad N=1,2, \ldots
$$

Из теоремы 4 следует, что если $a_{N}>0$ или $b_{N}<0$ для всех $N=1,2, \ldots$ и $\lim _{N \rightarrow \infty} b_{N} / a_{N}=1$, то последовательность случайных величин $2^{N}(N !)^{-1}\left(a_{N}+b_{N}\right)^{-N} \times$ $\operatorname{per} C, N=1,2, \ldots$, сходится по вероятности к единище. Если же $a_{N}<0<b_{N}$ и $a_{N}+b_{N} \neq 0$, то закон больших чисел для этой последовательности не выполняется.

Наконец, если $a_{N}=-b_{N}<0$, то

$$
\mathbf{E} \operatorname{per} C=0, \quad \mathbf{E}(\operatorname{per} C)^{2}=N !\left(a_{N}^{2} / 3\right)^{N}, \quad N=1,2, \ldots,
$$

и поэтому, если $a_{N} \rightarrow 0$ при $N \rightarrow \infty$ так, что $N !\left(a_{N}^{2} / 3\right)^{N} \rightarrow 0$, то последовательность per $C, N=1,2, \ldots$, сходится по вероятности к нулю.

Возвращаясь к общему случаю, заметим, что аналогичный подход применим к ситуации, когда рассматривается класс случайных матриц вида $D=\left(d_{i j}\right), i, j=1, \ldots, N$, в предположении, что строки матрицы $D$ суть независимые $N$-мерные случайные векторы, имеющие одинаковое распределение, совпадающее с распределением случайного вектора $\left(\mu_{1}, \ldots, \mu_{N}\right)$, компоненты которого также одинаково распределены, но, вообще говоря, зависимы. Будем предполагать, что моменты $\mathrm{E} \mu_{1} \mu_{2}$ и $\mathrm{E} \mu_{1}^{2}$ существуют. При этих условиях аналогично (6) получаем, что

$$
\mathrm{E}(\operatorname{per} D)^{2}=N ! \sum_{m=0}^{N}(N)_{m} S_{m}\left(\mathrm{E} \mu_{1} \mu_{2}\right)^{m}\left(\mathrm{E} \mu_{1}^{2}\right)^{M-m}=N !\left(\mathrm{E} \mu_{1}^{2}\right)^{N} \sum_{m=0}^{N}(N)_{m} S_{m} p^{m},
$$


где $p=\mathrm{E} \mu_{1} \mu_{2}\left(\mathbf{E} \mu_{1}^{2}\right)^{-1}$ и дополнительно предполагается, что здесь и далее в аналогичных случаях $\mathrm{E} \mu_{i} \mu_{j}=\mathrm{E} \mu_{1} \mu_{2}$ для всех $i \neq j, i, j=1,2, \ldots, N$.

Если, кроме того, $\mathrm{E} \mu_{1} \mu_{2} \neq 0$, то согласно (12)

$$
\mathbf{E}(\operatorname{per} D)^{2}=(N !)^{2}\left(\mathbf{E} \mu_{1} \mu_{2}\right)^{N} \sum_{m=0}^{N} \frac{\gamma^{m}}{m !},
$$

где $\gamma=\mathbf{E} \mu_{1}^{2}\left(\mathbf{E} \mu_{1} \mu_{2}\right)^{-1}-1$

Рассматривая схему серий, когда распределение случайного вектора $\left(\mu_{1}, \ldots, \mu_{N}\right)$ зависит от $N$, мы введем обозначения $\mu_{i}=\mu_{i N}, i=1, \ldots, N$, и будем предполагать, что

$$
\mathbf{E} \mu_{1 N}^{2}>0, \quad \mathbf{E} \mu_{1 N} \mu_{2 N} \neq 0, \quad N=1,2, \ldots,
$$

В этих предположениях находим, что

$$
\mathbf{E} \text { per } D=N !\left(\mathbf{E} \mu_{1 N}\right)^{N}, \quad \mathbf{E}(\operatorname{per} D)^{2}=(N !)^{-2}\left(\mathbf{E} \mu_{1 N} \mu_{2 N}\right)^{N} \sum_{m=0}^{N} \frac{\gamma_{N}^{m}}{m !}
$$

где

$$
\gamma_{N}=\mathbf{E} \mu_{1 N}^{2}\left(\mathbf{E}\left(\mu_{1 N} \mu_{2 N}\right)^{-1}-1, \quad N=1,2, \ldots\right.
$$

Таким образом, при условии, что $\mathbf{E} \mu_{1 N} \neq 0, N=1,2, \ldots$, получаем равенство

$$
\mathbf{E}(\operatorname{per} D)^{2}=(\mathbf{E} \operatorname{per} D)^{2}\left(\mathbf{E} \mu_{1 N} \mu_{2 N}\right)^{N}\left(\mathbf{E} \mu_{! N}\right)^{-2 N} \sum_{m=0}^{N} \frac{\gamma_{N}^{m}}{m !} .
$$

Отсюда следует, что справедливо следующее утверждение.

Tеорема 5. Пусть $\mathrm{E} \mu_{1 N} \neq 0, \mathbf{E} \mu_{1 N} \mu_{2 N} \neq 0, N=1,2, \ldots$, u npu $N \rightarrow \infty$

$$
\sum_{m=0}^{N} \frac{\gamma_{N}^{m}}{m !}=\left(\mathbf{E} \mu_{1 N}\right)^{2 N}\left(\mathbf{E} \mu_{1 N} \mu_{2 N}\right)^{-N}(1+o(1))
$$

где величина $\gamma_{N}$ определяется соотночением (37). Тогда последовательность случайных величин $(N !)^{-1}\left(\mathrm{E} \mu_{1 N}\right)^{-N} \operatorname{per} D, N=1,2, \ldots$, сходится по вероятности к единиче.

Следствие 1. Если Е $\mu_{1 N} \neq 0, \mathbf{E} \mu_{1 N} \mu_{2 N}>0, N=1,2, \ldots$, u $п и, N \rightarrow \infty$

$$
\begin{aligned}
\mathrm{E} \mu_{1 N}^{2} & \sim \mathrm{E} \mu_{1 N} \mu_{2 N}, \\
\left(\mathrm{E} \mu_{1 N} \mu_{2 N}\right)^{N} & \sim\left(\mathrm{E} \mu_{1 N}\right)^{2 N},
\end{aligned}
$$

то справедливо утверждение теоремы 5.

Замечание 13. Утверждение следствия 1 дает лишь достаточные условия справедливости закона больших чисел для последовательности

$$
(\text { E per } D)^{-1} \operatorname{per} D, \quad N=1,2, \ldots
$$

Так, например, в рассмотренной выше ситуации, когда вектор $\left(\mu_{1 N}, \ldots, \mu_{N N}\right)$ совпадает с вектором $\left(\xi_{1}, \ldots, \xi_{N}\right)$, из следствия 1 получаем, что закон больших чисел имеет место, если $n, N \rightarrow \infty$ так, что $N=o(n)$ (что слабее утверждения теоремы 1 ). Если же $\left(\mu_{1 N}, \ldots, \mu_{N N}\right)=\left(\varkappa_{1}, \ldots, \varkappa_{N}\right)$ (как в теореме 2$)$, то при $N \rightarrow \infty$ ни одно из условий (39), (40) не может выполняться. В то же время нетрудно проверить, что при выполнении условий теорем 1 или 2 оценка (38) справедлива. 
Теорема 6. Пусть $\mathrm{E} \mu_{1 N} \neq 0, \mathbf{E} \mu_{1 N} \mu_{2 N}>0, N=1,2, \ldots$, и пусть последовательность $\left\{\gamma_{N}\right\}$ ограничена или $\gamma_{N} \rightarrow \infty$ при $N \rightarrow \infty$ так, что $\left(N-\gamma_{N}\right) / \sqrt{\gamma_{N}} \rightarrow \infty$. Если, кроме того, при $N \rightarrow \infty$

$$
\gamma_{N} N^{-1}=2 \ln \left|\mathbf{E} \mu_{1 N}\right|-\ln \mathbf{E} \mu_{1 N} \mu_{2 N}+o(1 / N),
$$

то справедливо утверждение теоремы 5.

Утверждение теоремы 5 также верно, если при $N \rightarrow \infty$ параметр $\gamma_{N} \rightarrow \infty$ так, что $\left(N-\gamma_{N}\right) / \sqrt{\gamma_{N}} \rightarrow x \in \mathbf{R} u$

$$
\gamma_{N} N^{-1}=2 \ln \left|\mathrm{E} \mu_{1 N}\right|-\ln \mathrm{E} \mu_{1 N} \mu_{2 N}-\frac{1}{N} \ln \Phi(x)+o(1 / N) .
$$

Доказательство. Можно утверждать, что аналогично (32)-(34) в условиях теоремы 6

$$
\sum_{m=0}^{N}(m !)^{-1} \gamma_{N}^{m}=e^{\gamma_{N}} \Phi(x)(1+o(1))
$$

где либо $x \in \mathbf{R}$, либо $x=+\infty$ и $\Phi(+\infty)=1$.

Следовательно, согласно (42)

$$
\gamma_{N} N^{-1}=N^{-1} \ln \left(\sum_{m=0}^{N} \frac{\gamma_{N}^{m}}{m !}\right)-\frac{1}{N} \ln \Phi(x)+o(1 / N) .
$$

Из оценок (41) и (43) следует (38). Теорема 6 доказана.

Замечание 14. Если Е $\mu_{1 N}=0, \mathrm{E} \mu_{1 N} \mu_{2 N}>0$ и при $N \rightarrow \infty$ выполнено условие (39), To

$$
\mathbf{E}(\operatorname{per} D)^{2}=(N !)^{2}\left(\mathbf{E} \mu_{1 N} \mu_{2 N}\right)^{N}(1+o(1)) .
$$

Если, кроме того, $(N !)^{2}\left(\mathrm{E} \mu_{1 N} \mu_{2 N}\right)^{N} \rightarrow 0$ при $N \rightarrow \infty$, то последовательность per $D$, $N=1,2, \ldots$, сходится по вероятности к нулю.

В случае, когда $\mathrm{E} \mu_{1 N}=\mathrm{E} \mu_{1 N} \mu_{2 N}=0$, справедливо равенство

$$
\mathbf{E}(\operatorname{per} D)^{2}=N !\left(\mu_{1 N}^{2}\right)^{N}, \quad N=1,2, \ldots,
$$

и если $N !\left(\mathrm{E}_{1 N}^{2}\right)^{N} \rightarrow 0$ при $N \rightarrow \infty$, то справедливо предыдущее утверждение.

Наконец, в случае, когда $\mathrm{E} \mu_{1 N} \mu_{2 N}=0$ и $\mathbf{E} \mu_{1 N} \neq 0$, справедливо равенство

$$
\mathrm{E}(\operatorname{per} D)^{2}=(\mathrm{E} \operatorname{per} D)^{2} \frac{\left(\mathrm{E} \mu_{1 N}^{2}\right)^{N}}{N !\left(\mathrm{E} \mu_{1 N}\right)^{2 N}}, \quad N=1,2, \ldots,
$$

и если при $N \rightarrow \infty$

$$
\left(\mathbf{E} \mu_{1 N}^{2}\right)^{N}\left(\mathbf{E} \mu_{1 N}\right)^{-2 N} \sim N !
$$

то есть

$$
\ln \left(\mathbf{E} \mu_{1 N}^{2}\right)-2 \ln \left|\mathbf{E} \mu_{1 N}\right|=\ln N-1+\frac{1}{2 N} \ln (2 \pi N)+o(1 / N),
$$

то справедливо утверждение теоремы 5.

Заметим, что использованный при доказательствах приведенных в работе утверждений подход, связанный с применением так называемого метода вторых моментов, использовался в задачах вероятностной комбинаторики в [6]. 


\section{Список литературы}

1. Сачков В. Н., Вероятностные методы в комбинаторном анализе. Наука, Москва, 1978.

2. Прохоров Ю. В., ред., Вероятность и математическая статистика. Энииклопедия. Большая Российская энциклопедия, Москва, 1999.

3. O’Neil P. E., Asymptotics in random (0, 1)-matrices. Proc. Amer. Math. Soc. (1970) 25, №2, 290-295.

4. O'Neil P. E., Asymptotics and random matrices with row-sum and column-sum restrictions. Bull. Amer. Math. Soc. (1969) 75, №6, 1276-1282.

5. Тимашев А. Н., Закон больших чисел для перманентов случайных стохастических матриц. Дискретная математика (1999) 11, №3, 91-98.

6. Эрдеш П., Спенсер Дж., Вероятностные методы в комбинаторике. Мир, Москва, 1976.

Статья поступила 16.10.2003. 